\title{
Incidence of Vascular Complications Arising from Anterior Spinal Surgery in the Thoraco-Lumbar Spine
}

\author{
Zdenek Klezl $^{1,2}$, Girish Nanjunda Swamy ${ }^{1}$, Thomas Vyskocil ${ }^{2}$, Jan Kryl ${ }^{2}$, Jan Stulik ${ }^{2}$ \\ ${ }^{I}$ Department of Trauma and Orthopaedics, Royal Derby Hospital, Derby, UK \\ ${ }^{2}$ Department of Spinal Surgery, Charles University Medical School, Prague, Czech Republic
}

\begin{abstract}
Study Design: Modern biomaterials and instrumentation have popularised surgery of the thoraco-lumbar spine through an anterior route. The advantage of anterior surgery is that it allows for a direct decompression of the compromised spinal canal. However, the potential for devastating long-term sequelae as a result of complications is high.

Purpose: The aim of this study was to give a general overview and identify the incidence of vascular complications.

Overview of Literature: There is limited literature describing the overall incidence and complications of anterior spinal surgery.

Methods: A retrospective review of a prospective database of 1,262 consecutive patients with anterior surgery over a twelve-year period.

Results: In our study, $1.58 \%(n=20)$ of patients suffered complications. Injury to a major vessel was encountered in $14(1.11 \%)$ cases, of which nine involved an injury to the common iliac vein. In six cases, the original procedure was abandoned due to a life-threatening vascular injury ( $\mathrm{n}=3$ ) and unfavourable anatomy $(\mathrm{n}=3)$.

Conclusions: The incidence of vascular and other complications in our study was relatively low. Nevertheless, the potential for devastating long-term sequelae as a result of complications remains high. A thorough knowledge and awareness of normal and abnormal anatomy should be gained before attempting such a procedure, and a vascular surgical assistance especially should be readily accessible. We believe use of access surgeons is mandatory in cases with difficult or aberrant anatomy.
\end{abstract}

Keywords: Anterior thoraco-lumbar approach; Complications; Vascular injury; Left iliac vein; External iliac vein

\section{Introduction}

Anterior spinal surgery is becoming more popular with the advent of modern biomaterials and instrumentation. With the development of instrumentation designed specifically for anterior approach, instrumented stabilisation can be performed at the same time. Varieties of anterior approaches have been described according to the level of and indication for surgery [1]. With the increased use of these approaches, complications inevitably arise [2]. To date however, there appears to be limited literature describing the overall incidence and complications of such surgeries [2-6]. The aim of this study was therefore to give a general overview and identify the incidence of complications from anterior spinal surgery in the thoracic and lumbar spine.

Received Jan 9, 2013; Revised Jun 24, 2013; Accepted Jul 23, 2013

Corresponding author: Girish Nanjunda Swamy

Department of Trauma and Orthopaedics, Royal Derby Hospital,

Uttoxeter Road, Derby DE22 3NE, UK

Tel: +44-7792034317, Fax: +44-1332787556, E-mail: girishjam@yahoo.com 
Table 1. Tables illustrating the number of patients treated with their surgical levels

\begin{tabular}{|c|c|c|c|}
\hline \multicolumn{2}{|c|}{ Discectomy } & \multicolumn{2}{|c|}{ Corpectomy } \\
\hline Segment & Number & Segment & Number \\
\hline $\mathrm{T} 1-2$ & 5 & $\mathrm{~T} 1$ & 6 \\
\hline T2-3 & 2 & $\mathrm{~T} 2$ & 7 \\
\hline T3-4 & 2 & T3 & 6 \\
\hline T4-5 & 3 & T4 & 13 \\
\hline T5-6 & 5 & T5 & 15 \\
\hline T6-7 & 6 & T6 & 15 \\
\hline T7-8 & 13 & $\mathrm{~T} 7$ & 15 \\
\hline T8-9 & 9 & T8 & 20 \\
\hline T9-10 & 10 & T9 & 21 \\
\hline T10-11 & 13 & $\mathrm{~T} 10$ & 20 \\
\hline T11-12 & 52 & $\mathrm{~T} 11$ & 24 \\
\hline T12-L1 & 116 & $\mathrm{~T} 12$ & 60 \\
\hline L1-2 & 66 & L1 & 96 \\
\hline L2-3 & 51 & L2 & 51 \\
\hline L3-4 & 63 & L3 & 42 \\
\hline L4-5 & 165 & L4 & 20 \\
\hline L5-S1 & 231 & L5 & 15 \\
\hline L5-6 & 3 & L6 & 0 \\
\hline L6-S2 & 1 & & \\
\hline
\end{tabular}

Table 2. Complications as a result of anterior spinal surgery to the thoracic and lumbar spines

\begin{tabular}{|cc|}
\hline \multicolumn{1}{|c}{ Injured structures } & Number \\
\hline Vasculature & \\
\hline Common iliac vein & 9 \\
\hline External iliac vein & 1 \\
\hline Inferior vena cava & 2 \\
\hline Arterial injury & 2 \\
\hline Total number & 14 \\
\hline Other complications included & \\
\hline Thoracic duct injury & 3 \\
\hline Ureteric injury & 2 \\
\hline Infection & 1 \\
\hline
\end{tabular}

\section{Materials and Methods}

A retrospective review of a prospectively collected database spanning twelve years (1998-2010), of all patients who underwent anterior spinal surgery of the thoracic and lumbar spines, was undertaken. Data regarding patient demographics, the level and type of surgery, indications for surgery and complications were obtained. During this period, there was no variation in the surgical protocol.

The surgical approach was standardised in all procedures: right-sided lateral thoracotomy for pathologies localized at T2-T6; and left lateral thoracotomy for the rest of the thoracic spine levels and the thoraco-lumbar junction. Lumbar spine was approached from the left side through a retroperitoneal approach, rarely from the right side. We preferred to use cages filled with autologous bone graft or cement in selected bone tumours. Where there was aberrant anatomy, for example in cases with a low bifurcation aorta and vena cava in patients undergoing anterior lumbar interbody fusion, an alternative interbody device was used, i.e., tricortical bone graft(s). A cell saver was used in the corpectomy procedures where posttraumatic correction was required. Intraoperative blood loss was analysed reviewing surgical and anaesthetic notes.

During the period, 1,262 patients underwent anterior spinal surgery at either thoracic or lumbar levels. Eight hundred and sixteen patients underwent discectomy with 231 cases at L5/S1 level. Four hundred and forty six patients underwent corpectomy, with 96 occurring at L1 level (Table 1, Figs. 1, 2). There were $42.23 \%$ of cases that underwent anterior surgery for trauma, and $27.97 \%$ for degenerative conditions. The remainder was for deformity correction (12.6\%), infection $(7.77 \%)$ and neoplasm (9.42\%).

There were 658 males. Age ranged from 7 to 92 years. The two senior authors (J.S. and Z.K.) performed all surgeries. A vascular surgeon was always available either in the operating theatre or within the hospital premises for any possible complications in all cases.

\section{Results}

In our study, $1.58 \%(\mathrm{n}=20)$ of patients suffered complications (Table 2). The majority of complications occurred with surgery performed at the lumbar level. Injury to a major vessel was encountered in $14(1.11 \%)$ cases, of which nine involved injury to the common iliac vein (Fig. 3). The inferior vena cava (IVC) was injured in two patients, and the external iliac vein was injured in one patient. These injuries occurred with a left-sided approach. The thoracic duct was injured on three occasions, identi- 
fied by the drainage of chyle. A repair was not deemed necessary, with the three patients recovering fully. In six cases, the original procedure was abandoned due to a life-threatening vascular injury $(\mathrm{n}=3)$ and unfavourable anatomy $(n=3)$. There was one case of superficial wound abscess which was healed by secondary intention. There were no patients with postoperative retrograde ejaculation. There were no documented cases of an ischaemia of the cord in patients with an anterior approach.

\section{Studies}

1) Case 1

In a 67-year-old patient with spondylodiscitis at L4-L5

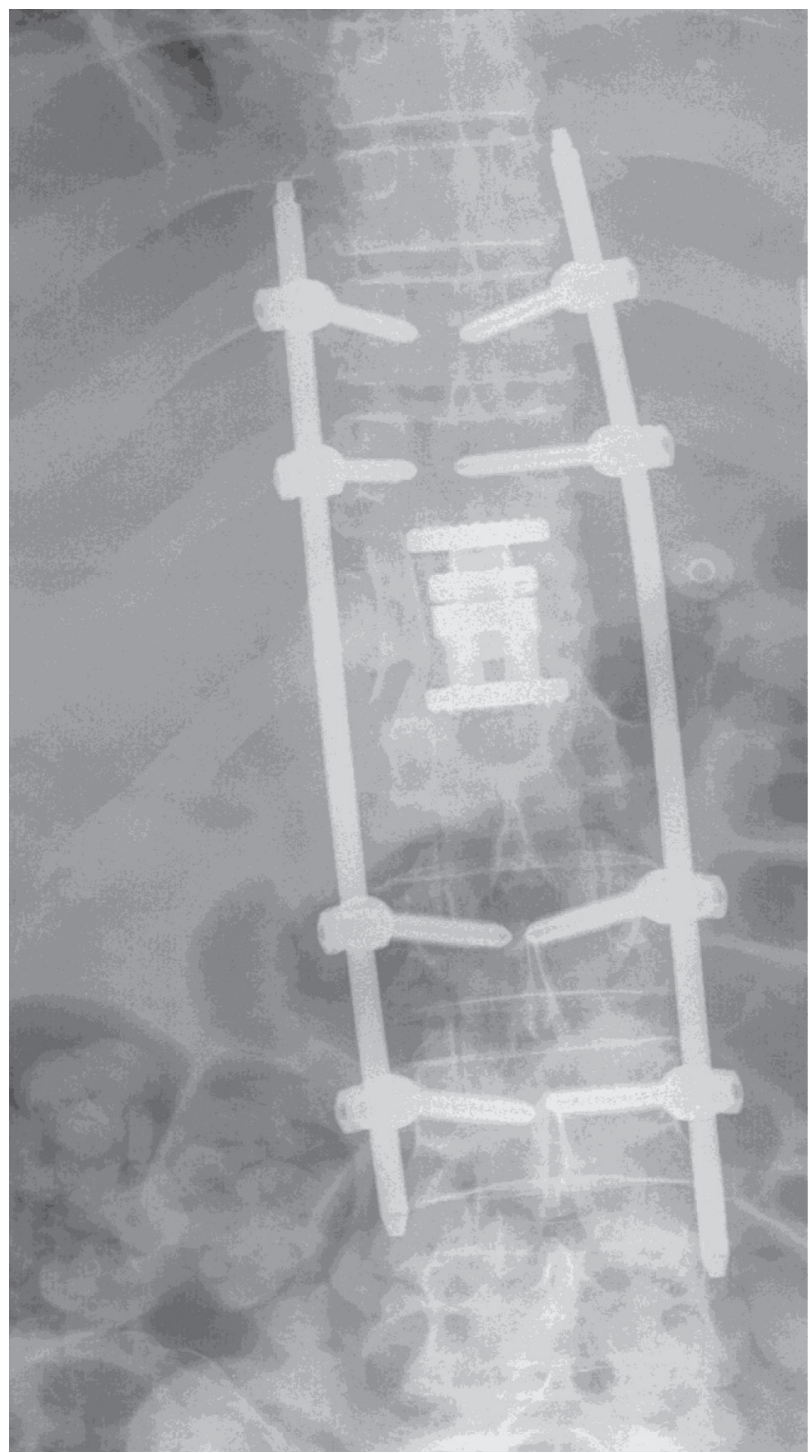

Fig. 1. An antero-posterior radiograph of a patient who underwent a 360 degree fusion with anterior corpectomy for trauma. level, during the anterior surgery, the left common iliac vein was accidentally injured by traction associated with mobilizing of the big vessels to the right side, which led to avulsion of the hidden ascending iliolumbar vein from the main trunk resulting in a 5-mm defect. The left ureter was transected as well due to presence of heavy adhesions. The vein was sutured as well as the ureter, which was fitted with a pigtail drain and introduced into the bladder. L4-L5 discectomy was carried out, and a cage with bone graft was inserted. Subsequently, urologists removed the ureter drain with cystoscopy. The patient's clinical outcome was not compromised.

\section{2) Case 2}

A 44-year-old woman was scheduled for 360-degree fusion for chronic low back pain at the L4-L5 level, using left-side retroperitoneal video-assisted approach. Once

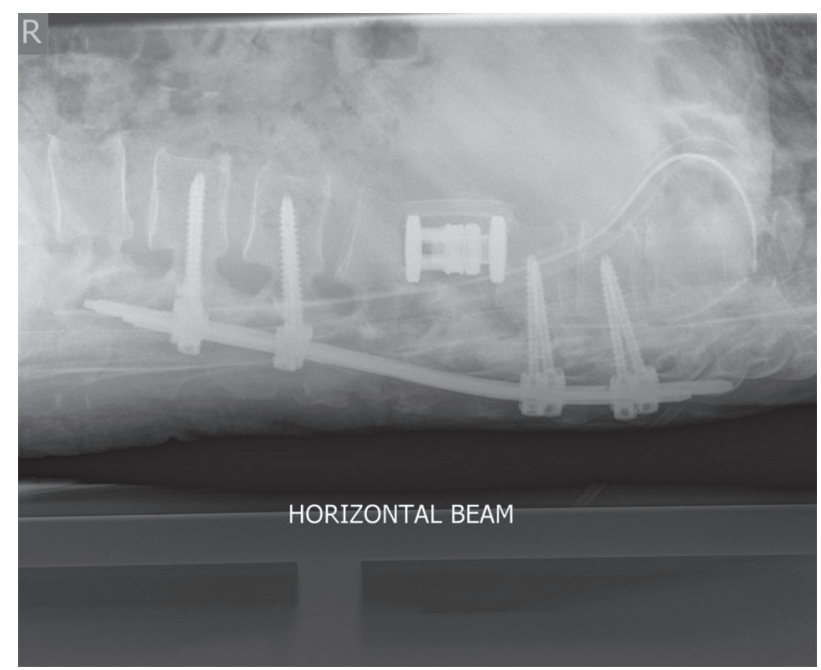

Fig. 2. A lateral radiograph of the same patient as in Fig. 1.

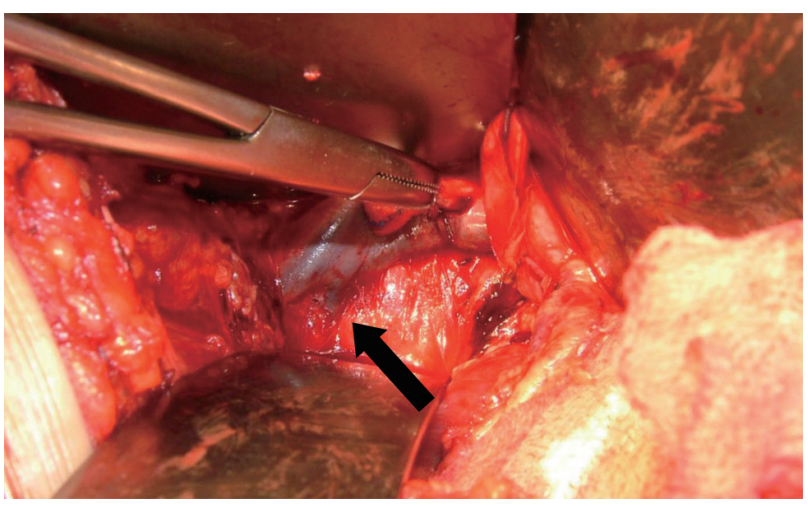

Fig. 3. Left common iliac vein with ascending iliolumbar vein which (arrow) can be avulsed once mobilizing of the big vessels is done to the right side. 
the exposure was done, a retractor was placed on the right side of the spin to retract the big vessels. Unfortunately, it was not recognized that the IVC was not retracted enough but only compressed by the retractor (Fig 4). The surgeon made the usual window-cut in the disc, cutting a $7 \mathrm{~mm} \times 3 \mathrm{~mm}$ defect in the IVC. Massive bleeding followed once the retractor changed position. Procedure was immediately converted into an open approach, and the assisting vascular surgeon repaired the defect. In a four-hour long surgery, discectomy and fusion were completed. Final check of the left common iliac artery revealed no pulsation, and the vessel was catheterized with Fogarty catheter; and flow was reinstated and defect sutured. The patient was then turned and underwent posterior translaminar screw fixation. She recovered from the surgery uneventfully and was discharged on the fourth postoperative day.

\section{Discussion}

In a review of 1,223 procedures with an anterior approach, Faciszewski et al. [7] reported a complication rate of $11.5 \%$. McDonnell et al. [2] reported a complication rate of $31 \%$ in the sample of 447 patients; and with the majority of cases being for deformity correction, a higher rate of complications was expected. Although this study included all patients undergoing surgery via an anterior approach and not only those who had fusion surgery, the

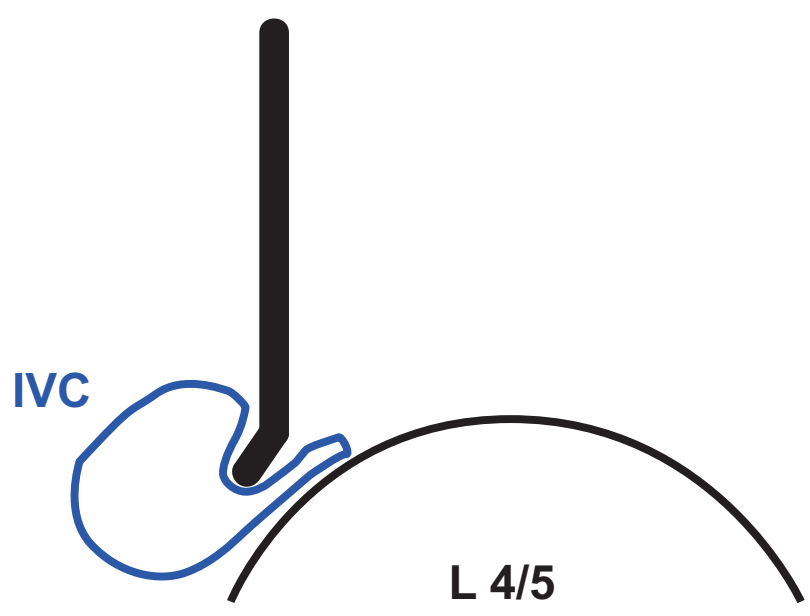

Fig. 4. Diagram illustrating suboptimally placed retractor which is compressing part of vena cava circumference and not safely retracting it. Incision of the annulus may then lead to significant injury of the vein. IVC, inferior vena cava. incidence of complications for non-deformity cases was small (0.75\%). Also, unlike earlier studies, the data in our study was obtained from a prospective database over a more recent time-frame with improved techniques. Our sample size is also much larger, allowing for a more accurate quantification of the incidence of complications.

The true incidence of vascular complications during anterior spinal surgery is unknown, as many do not result in significant clinical impairment, though some studies have quoted rates of $0.08 \%$ to $18.4 \%[5,7,8]$. Such variability can be explained by the degree of scarring or adhesions at the operated levels, the type of incision for the anterior approach used and the indications for surgery. For example, deformity surgery would result in higher risks of neurovascular complications, particularly if there was an underlying intraneural pathology or aberrant anatomy [9].

More recent studies by Brau et al. [3,4] have reported a vascular complication rate of $1.9 \%$ in a large cohort of 1,310 anterior lumbar surgeries. The report concluded that anterior lumbar surgery is safe but must be carried out with the utmost care for the vessels, to avoid a possible catastrophic problem.

A recent retrospective study on 338 patients has revealed the incidence of major vascular complications following anterior lumbar surgery to be $2.9 \%$ [6]. The major vessel commonly injured was the common iliac vein, with the left side being more common at the L5/S1 level. All right common iliac vein injury was often seen at the L4/5 level. Patients who have had previous osteomyelitis/discitis, previous anterior spinal surgery, anatomical anomalies (e.g., spondylolisthesis) and transitional vertebrae were at higher risks of potential major vessel injuries. The present study did not demonstrate any injuries to the common iliac arteries [10]; however there were four cases of injury to the iliac veins. This finding is in keeping with previous studies. A possible explanation for this is the friability of the vein walls, rendering them more susceptible to injury by extraneous placement of retractors.

The blood supply to the lower half of the spinal cord comes from the artery of Adamkiewicz and may be located anteriorly at any level from T5 to L2. In majority of cases, however, this artery arises on the left side between T9-T12. Although injury to this vessel is often tolerated, its identification and preservation should be done to prevent potential devastating sequelae. A recent study [11] has demonstrated that spinal cord ischaemia occurs when 
both arteries are injured if the surgery is conducted on more than four levels including the level at which the artery of Adamkiewicz arises. We have been fortunate and have not encountered any arterial injuries to the cord via the anterior approach by the demonstration of no cases of an ischaemic cord.

Leakage of contents from the thoracic duct (chylothorax) is a rare complication in the retroperitonal approach to the anterior spine, with the thoracolumbar region being the most at risk. In these cases, access to the upper lumbar/ thoracic spine is achieved by the taking-down of the diaphragm. Management of such cases is conservative, with the majority spontaneously stopping [12]. This regime was adopted for the two cases of thoracic duct injury encountered in this study. Neither case resulted in long-term sequelae as a result of the injury not being repaired.

Ureteric injury is a recognised problem that can occur following anterior exposure of the spine. The true incidence is unknown.

\section{Conclusions}

The advantages of anterior surgery for fractures of the thoracolumbar spine are made more apparent by the fact that it allows for a direct decompression of the compromised spinal canal. However, although the incidence of complications is relatively low in this study, the potential for devastating long term sequelae is high. A thorough knowledge and awareness of normal and abnormal anatomy should be gained before attempting such procedures. In patients with tumours, infection, previous irradiation or anticipated difficult anatomy, we feel that the attendance of a vascular surgeon is mandatory.

\section{Conflict of Interest}

No potential conflict of interest relevant to this article was reported.

\section{References}

1. Gumbs AA, Bloom ND, Bitan FD, Hanan SH. Open anterior approaches for lumbar spine procedures.
Am J Surg 2007;194:98-102.

2. McDonnell MF, Glassman SD, Dimar JR 2nd, Puno RM, Johnson JR. Perioperative complications of anterior procedures on the spine. J Bone Joint Surg Am 1996;78:839-47.

3. Brau SA, Delamarter RB, Schiffman ML, Williams LA, Watkins RG. Vascular injury during anterior lumbar surgery. Spine J 2004;4:409-12.

4. Brau SA, Delamarter RB, Schiffman ML, Williams LA, Watkins RG. Left iliac artery thrombosis during anterior lumbar surgery. Ann Vasc Surg 2004;18:4851.

5. Baker JK, Reardon PR, Reardon MJ, Heggeness MH. Vascular injury in anterior lumbar surgery. Spine (Phila Pa 1976) 1993;18:2227-30.

6. Fantini GA, Pappou IP, Girardi FP, Sandhu HS, Cammisa FP Jr. Major vascular injury during anterior lumbar spinal surgery: incidence, risk factors, and management. Spine (Phila Pa 1976) 2007;32:2751-8.

7. Faciszewski T, Winter RB, Lonstein JE, Denis F, Johnson L. The surgical and medical perioperative complications of anterior spinal fusion surgery in the thoracic and lumbar spine in adults: a review of 1223 procedures. Spine (Phila Pa 1976) 1995;20:1592-9.

8. Westfall SH, Akbarnia BA, Merenda JT, et al. Exposure of the anterior spine. Technique, complications, and results in 85 patients. Am J Surg 1987;154:700-4.

9. Leung YL, Grevitt M, Henderson L, Smith J. Cord monitoring changes and segmental vessel ligation in the "at risk" cord during anterior spinal deformity surgery. Spine (Phila Pa 1976) 2005;30:1870-4.

10. Raskas DS, Delamarter RB. Occlusion of the left iliac artery after retroperitoneal exposure of the spine. Clin Orthop Relat Res 1997;(388):86-9.

11. Kato S, Kawahara N, Tomita K, Murakami H, Demura S, Fujimaki Y. Effects on spinal cord blood flow and neurologic function secondary to interruption of bilateral segmental arteries which supply the artery of Adamkiewicz: an experimental study using a dog model. Spine (Phila Pa 1976) 2008;33:1533-41.

12. Su IC, Chen CM. Spontaneous healing of retroperitoneal chylous leakage following anterior lumbar spinal surgery: a case report and literature review. Eur Spine J 2007;16 Suppl 3:332-7. 